\title{
3D Finite Element Simulation from Non-destructive X-ray Tomography and Verification with Novel Mechanical Testing and Digital Image Correlation In-situ of Focused Beam Microscope
}

\author{
Joseph Favata $^{1}$, S. Ali Shojaee ${ }^{2}$ and Sina Shahbazmohamadi ${ }^{3}$ \\ ${ }^{1}$ University of Connecticut, Willington, Connecticut, United States, ${ }^{2}$ Thermofisher Scientific, Austin, \\ Texas, United States, ${ }^{3}$ REFINE lab/ UCONN, Storrs, Connecticut, United States
}

Every electric-powered system whether it is a computer, phone, car, or clock possesses at least one packaged micro- or nano-electronic device. Traditional methods of mechanical quality assurance for packaged devices are outlined in standards such as MIL STD 883, SAE AS6171/5, among others and are primarily destructive in nature. For a device with a bond wire interconnect architecture, which account for roughly $90 \%$ of circulated devices [1-6] the device's packaging is removed and the bond wires are pulled until failure before a statistical inference is made on whether to accept the device that will ultimately be integrated. Among other shortcomings, this practice introduces uncertainty since the device that is being integrated is different from the tested device. A non-destructive testing alternative centered around the device that will be integrated has been shown for a bond wire pull test [7]. In summary, realistic geometries of the internal components of the device are captured with 3D X-ray tomography, segmented, reverse engineered to a CAD-editable format, and brought to finite element modeling to perform the mechanical test in simulation space. The advantages of this non-destructive approach include: reusability and assurance of the tested circuit that will be integrated, simultaneous testing of multiple components under different conditions, testing bond wires with fine pitch/shared leads, significantly faster testing in long duration dynamic tests (e.g. fatigue, creep, etc.), creating extremely unique test environments that could be prohibitively expensive to recreate (e.g. extreme pressure, extreme temperature, etc.), and performing parametric studies to answer design questions shortcutting the iterative prototyping process [7]. The results of this testing alternative requires sufficient validation based in physical testing. In this study, a novel in-situ mechanical tester was developed and used to perform the bond wire mechanical test in-situ of a FIB-SEM system. The data gathered from this physical testing was used to find the physical principal strains of the bond wire through digital image correlation (DIC). These principal strain values were used as validation criteria for the simulation models developed with the non-destructive workflow.

The device selected for this study was a QFP, single-die architecture part from Retail Alliance Limited (Part No.:A-4.0000-15). This model was selected randomly to illustrate that the methodology is indiscriminating. The circuit's tomography data was acquired with a Zeiss 510 Versa (Pleasanton, CA). The tomography parameters included $150 \mathrm{kV}-10 \mathrm{~W}$ beam settings, an acquisition pixel size of $3.74 \mu \mathrm{m}$ and camera binning of 2 . The images were acquired over $180^{\circ}$ of rotation and 1600 projections with a total acquisition time of about 3.5 hours. Certain steps were taken to optimize the tomography that are outlined in other publications [8-9]. The image segmentation was performed in Thermofisher Scientific Avizo Fire 8.1 (Hillsboro, OR). Greyscale thresholding was utilized to segregate voxel values unique to the bond wires. The small lead-frame stitch bond was segmented manually with a pixel paintbrush tool. The voxels were isotopic and cubic with dimensions of the pixel size before conservative smoothing was applied. The reverse engineering to a CAD-editable format was carried out in ANSYS SpaceClaim 17.1 (Canonsburg, PA) and the simulation was performed with COMSOL Multiphysics 5.2 (Burlington, MA) with linear elastic and linear elastoplastic constitutive models, though the procedure can be carried out on any reverse engineering CAD and multiphysics FEA software package [7]. The results of the simulation are shown in 
Figure 1. The location of consequential strain was found near the ball bond. The novel mechanical tester capable of performing the mechanical test in-situ of a focused beam microscope was used to perform the bond wire pull test while the area near the ball bond was imaged by SEM [10]. Selected snapshots along the loading path of the in-situ bond wire pull test are shown in Figure 1. The images were used as inputs to a digital image correlation algorithm developed within Thermofisher Scientific Avizo 2019.3 (Hillsboro, OR). A local subset-based DIC algorithm was leveraged where individual elements of the image data are independently correlated to each other and there is no field-continuity. Figure 2 displays the cumulative strain maps and locations of probe lines where strain values were compared among the FEdatasets and physical DIC dataset. The cumulative principal strain values were compared to the cumulative principal strain values of the finite element simulation results when utilizing linear elastic and linear elastoplastic constitutive models. The principal strain values along the line probe closely match across the simulation and physical tests except for the unrealistic drop in strain seen in the physical test around $15000 \mathrm{~nm}$. This was the result of filtering a data point with low correlation confidence. Figure 2 also displays the global behavior of the wire in the model and its relationship with the behavior of the wire from physical tests. The bond wire displaced and deformed along the loading curve similarly in simulation space as in the physical tests. Discrepancies at the lower values of loading curve were attributed to bond wire sag in the physical tests. Consequential loading behavior in the non-linear region was captured well. In both graphs, the more expensive linear elastoplastic constitutive model held a tighter correlation to the physical tests. With validation from the physical tests, the simulation model can be used to nondestructively test against the traditional acceptance criteria from published standards and since the geometry is CAD-editable, can be used for parametric design studies to address economically consequential questions associated with wire material, diameter, and shape with little effort. 

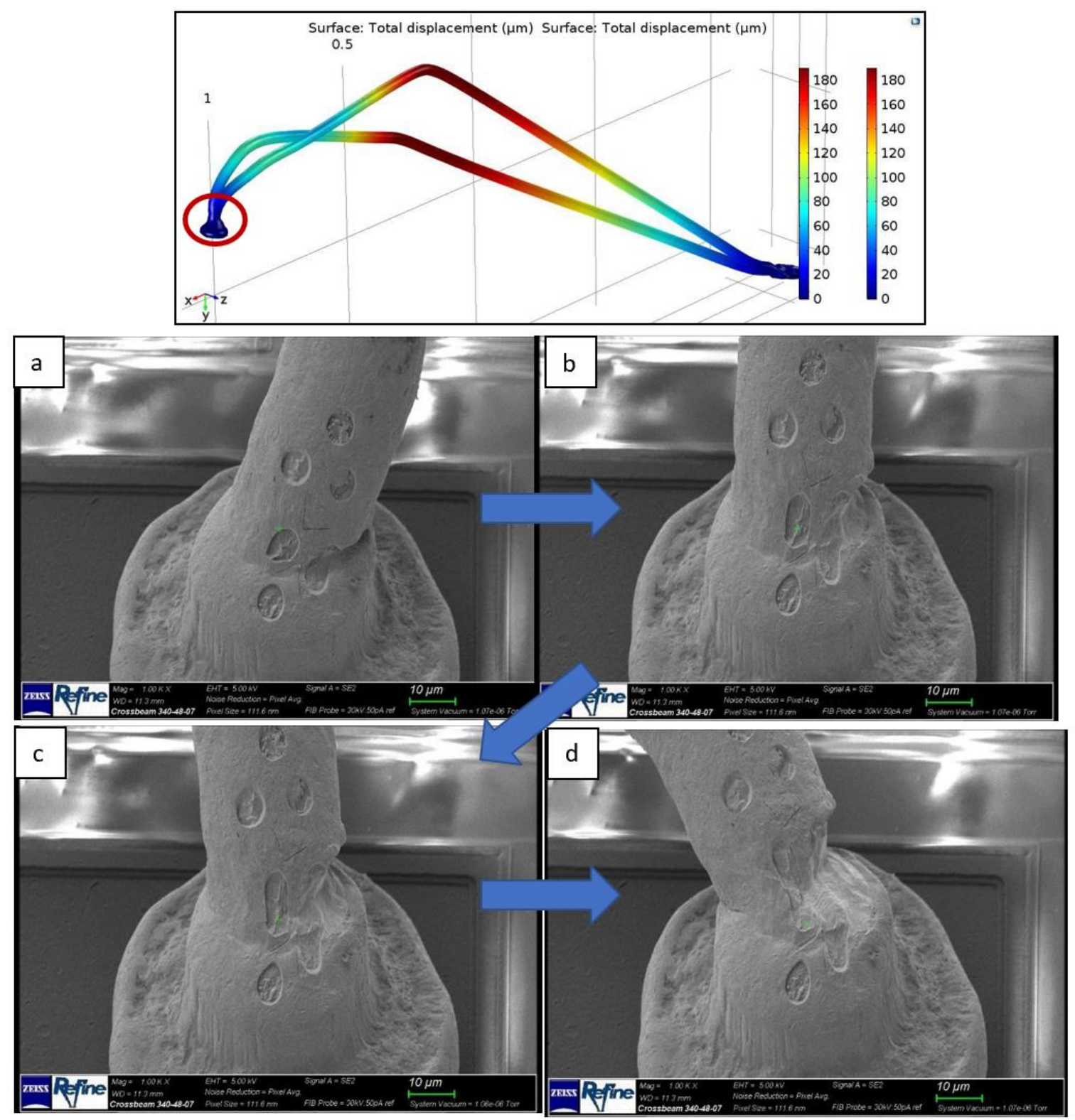

Figure 1. Bond wire geometries before and after loading within finite element modeling with total displacement map applied to both geometries (top). Chronological SEM images taken throughout in-situ bond wire pull test near ball bond- (a) Undeformed bond wire with multiple fiducial marks for DIC tracking, (b) and (c) rigid-body and deformable motion of bond wire under test, and (d) major plastic deformation and necking at failure site of bond wire [11]. 


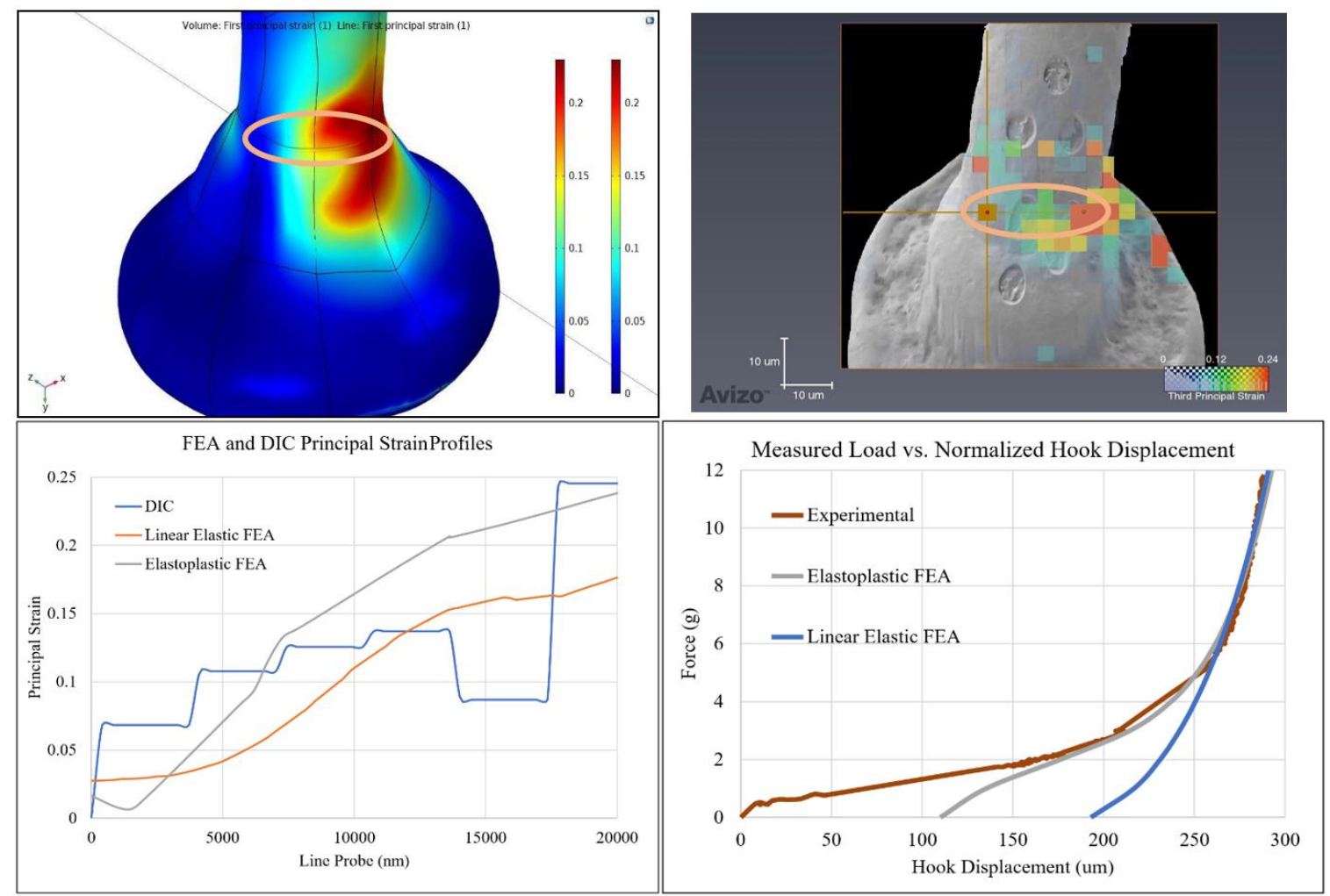

Figure 2. Line profile of principal strain on cumulative principal strain map from FEA (top left). Line profile of principal strain on cumulative principal strain map from DIC marked with two pin-point dots to mark the start and end of probe (top right). Principal strain measurements along line probes of DIC and FEA datasets (bottom left). Mechanical response of bond wire from experiment and FEA datasets - Force is the measured reaction force from the bond wire and hook displacement is the vertical displacement of the bond wire-hook interaction zone along the loading path (bottom right) [11].

\section{References}

[1] S. Schindler and K. W. M. Wohnig, "Development of a fine pitch copper wire bond process for integrated circuit devices," in 31st International Spring Seminar on Electronics Technology (ISSE), Budapest, Hungary, 2008.

[2] G. Harman, Wire Bonding in Microelectronics, 3rd ed., New York: McGraw-Hill, 2010.

[3] M. Roma, "Interconnect Mechanisms in Microelectronic Packaging," ProQuest Dissertations Publishing, Binghampton, 2016.

[4] G. Zhang, "Reliability of wire bonds in micro-electronic packages," Microelectronics International, vol. 25, no. 2, pp. 15-22, 2008.

[5] G. C. Leong and H. Uda, "Comparitive reliability studies and analysis of $\mathrm{Au}, \mathrm{Pd}-$ coated $\mathrm{Cu}$ and $\mathrm{Pd}-$ doped $\mathrm{Cu}$ wire in microelectronics packaging," PLOS ONE, vol. 8, no. 11, 2013.

[6] W. v. Driel, R. V. Silfhout and G. Zhang, "On wire failures in microelectronic packages," IEEE Transactions on Device and Materials Reliability, vol. 9, no. 1, pp. 2-8, 2009.

[7] J. Favata and S. Shahbazmohamadi, "Realistic on-destructive testing of integrated circuit bond wiring using 3-D X-ray tomography, reverse engineering, and finite element analysis," Microelectronics Reliability, vol. 83, pp. 91-100, 2018. 
[8] Shahbazmohamadi, D. Forte and M. Tehranipoor, "Advanced Physical Inspection Methods for Counterfeit IC Detection," in Conference Proceedings from the $40^{\text {th }}$ International Symposium for Testing and Failure Analysis (ISTFA), Houston, 2014.

[9] K. Mahmood, P. Carmona, S. Shahbazmohamadi, F. Pla and B. Javidi, "Real-time automated counterfeit integrated circuit detection using X-ray microscopy," Applied Optics, vol. 54, no. 13, pp. D25D32, 2015.

[10] J. Favata, S. Shahbazmohamadi, V. Ray and A. Hadjikhani, "Real-time, direct measurement mechanical tester in-situ of scanning beam microscope". Patent Application Number 16/569,384, (Patent Pending).

[11] Favata, Joseph, "Correlative Framework of Techniques for the Inspection, Evaluation, and Design of Micro-electronic Devices" (2020). Doctoral Dissertations. 2402. 